\title{
Inflammatory breast carcinoma
}

\author{
Kelli Y. Ha, MD, Shannon B. Glass, MD, and Louba Laurie, MD
}

Inflammatory breast carcinoma is a rare form of invasive breast cancer often characterized by erythema, warmth, and a classic "peau de orange" or "orange peel" appearance of the affected breast. The average age of onset is within the fourth and fifth decades. Lesions are usually detected and evaluated with mammography, sonography, and recently, breast magnetic resonance imaging. We present the case of a 49-year-old woman with inflammatory breast carcinoma in her left breast and describe the imaging appearance of this aggressive lesion on the modalities listed above. Because this lesion may be misdiagnosed as infection (i.e., mastitis) or as the sequelae of a dermatologic disorder, proper characterization of inflammatory breast carcinoma is of the utmost clinical and radiologic importance.

\section{CASE REPORT}

A 49-year-old woman presented with a history of a palpable mass within her left breast, which had been present for 2 years. Routine digital mammographic imaging of the right breast (not shown) did not demonstrate any abnormality. Within the upper outer aspect of the left breast at the palpable area of concern, a region of asymmetry, partially obscured by coarse trabecular thickening, was seen in addition to marked left axillary lymphadenopathy (Figure 1). Diffuse cutaneous thickening was also demonstrated within the left breast. Sonography demonstrated a large heterogeneous mass at the palpable area of concern, in the 2:00 position, $10 \mathrm{~cm}$ from the nipple, measuring approximately $4.6 \times 4.5 \times 4.4 \mathrm{~cm}$ (Figure 2). Left axillary lymphadenopathy, measuring up to $4.4 \mathrm{~cm}$ in the long axis, was also observed. The patient underwent surgical biopsy, and the histology proved consistent with inflammatory breast carcinoma (IBC). She was subsequently treated with a neoadjuvant chemotherapy regimen consisting of six cycles of 5-fluorouracil, doxorubicin, and cyclophosphamide. Mastectomy was then performed.

\section{DISCUSSION}

IBC is an infrequent form of invasive breast cancer that often presents with a rapid onset of diffuse erythema, warmth, edema, and/or peau d'orange changes of the breast. In addition, these changes are accompanied by breast tenderness and/or pain, palpable mass or masses, and diffuse rapid breast enlargement in conjunction with cutaneous thickening and dermal ridging. In the absence of clinical symptoms and abnormal laboratory values, i.e., fever, chills, and/or an elevated white blood cell count, one should immediately consider a diagnosis of IBC. This disease entity accounts for $1 \%$ to $6 \%$ of all breast cancers in the United States, with the average age of onset between 45 and 54 years (1-3). Similar to other types of breast carcinoma, IBC can occur in men, but usually at an older age (median age at diagnosis 66.5 years) than in women. IBC cases in men constitute between $0.6 \%$ and $1.4 \%$ of all newly diagnosed breast cancers (4).

Pathologic diagnosis of this clinical entity is generally based upon the presence of tumor emboli within dilated lymphatic vessels and a surrounding lymphocytic reaction in the dermis (2, 5; Figure 3). Although infiltration of dermal lymphatics confirms a diagnosis of IBC, the disease may only be evident clinically, and punch biopsies may be negative. In such cases, patients are treated for IBC based upon clinical data. While IBC is relatively uncommon compared with other malignancies, it remains aggressive, with a tendency to metastasize early (3). Therefore, information about this malignancy is of interest and importance to both radiologists and clinicians alike.

On mammography, IBC demonstrates a pattern of diffuse "inflammatory change," to include cutaneous and trabecular thickening, increased parenchymal density, breast distortion, and nipple retraction. Changes on mammography are secondary to dermal lymphatic infiltration and obstruction by tumor, rather than a true inflammatory process. Because of the increased parenchymal density, which is often seen throughout the affected breast, a focal mass lesion or group of suspicious calcifications is less often seen mammographically $(2,6)$. Additional associated mammographic findings, albeit observed less consistently, include axillary lymphadenopathy and nipple retraction. Of particular note, the contralateral breast should be carefully inspected for developing asymmetry and trabecular and/or cutaneous thickening, given the possibility of cancer spread or a simultaneous lesion in the contralateral breast, which occurs in approximately $1 \%$ to $5 \%$ of patients with primary IBC (5).

From the Department of Radiology, Baylor University Medical Center at Dallas. Corresponding author: Kelli Y. Ha, MD, Department of Radiology, Baylor University Medical Center at Dallas, 3500 Gaston Avenue, Dallas, TX 75246 (e-mail: yeek816@gmail.com). 

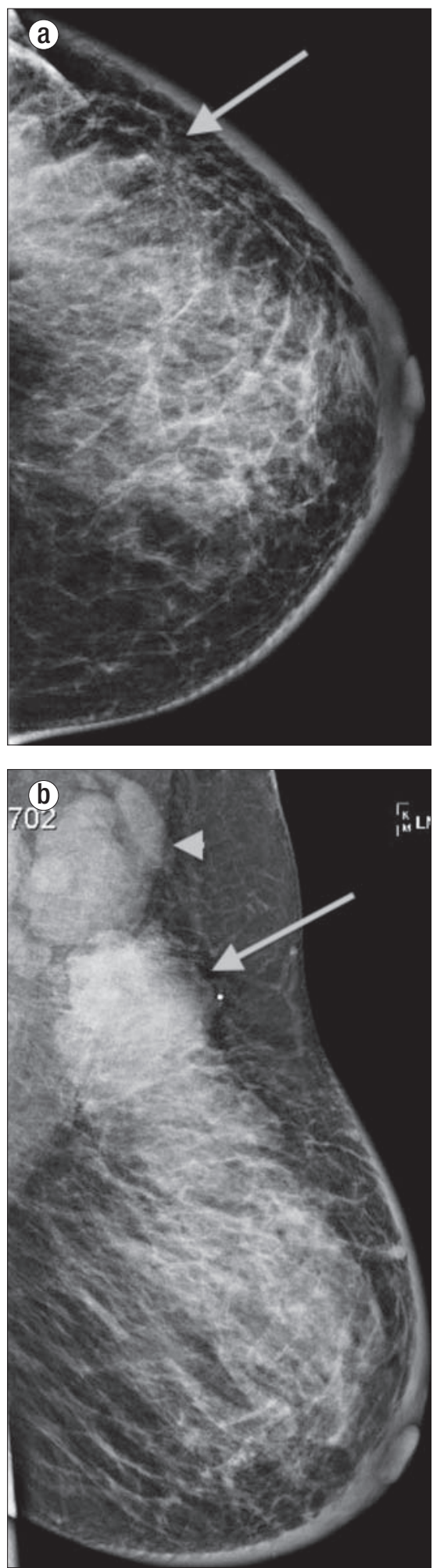

Figure 1. Routine digital mammographic imaging of the left breast: (a) craniocaudal view, (b) mediolateral view. Both views demonstrate a region of asymmetry (arrow), which corresponds to the palpable area of concern in the upper outer quadrant. Diffuse cutaneous and trabecular thickening is also identified about the surface of the left breast, which gives the appearance of increased breast density.
IBC may be divided into two clinical subgroups, first described by Taylor and Meltzer in 1938. The primary form is seen in patients with IBC characteristics that are evident from the onset, while the secondary form is described in those whose clinical features appear subsequent to appropriate treatment of a primary noninflammatory breast carcinoma (7). Given that our current patient presented without a known history of previously treated breast carcinoma, her disease was characterized as a primary lesion.

Sonographic evaluation of IBC is helpful in demonstrating lymphatic dilatation in association with tumor emboli, as well as depiction of hypoechoic shadowing masses, cutaneous thickening, skin/pectoral muscle invasion, and axillary lymphadenopathy. Sonography is notably a better modality for depicting these characteristics and should be used in conjunction with mammography and physical examination for diagnosis whenever possible. Sentinel node biopsy is ultimately used for the diagnosis of axillary involvement, as skin punch biopsy or ultrasound-guided core needle biopsy of suspicious masses is for IBC (3).

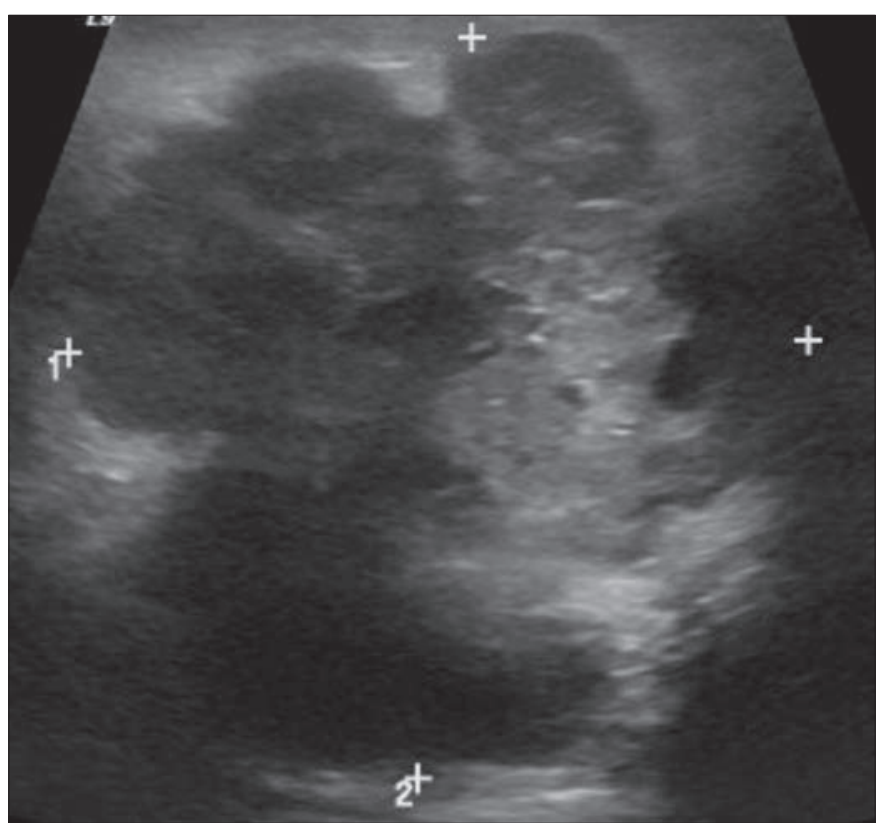

Figure 2. Sonographic image of the left breast demonstrates a large heterogeneous, predominantly hypoechoic mass at the 2:00 position of the left breast, $10 \mathrm{~cm}$ from the nipple, measuring approximately $4.6 \times 4.5 \times 4.4 \mathrm{~cm}$.

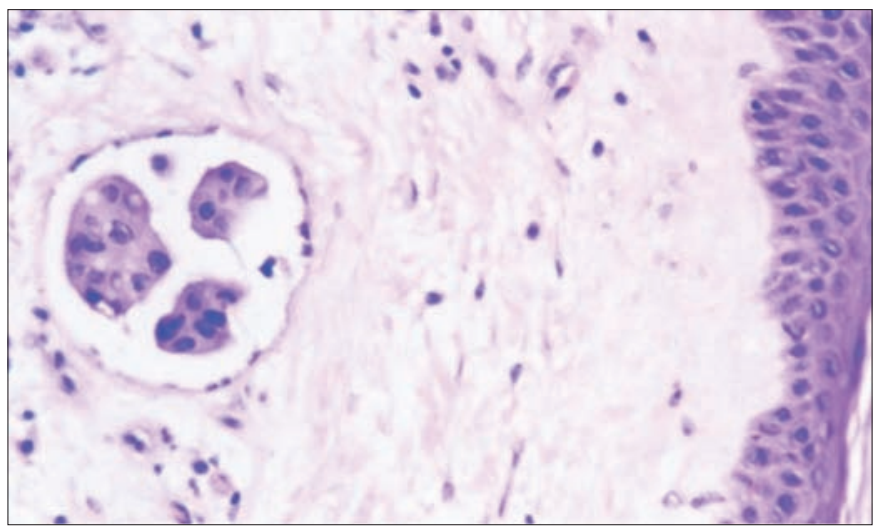

Figure 3. An example of inflammatory breast carcinoma (taken from a different patient) with a characteristic tumor embolus in a dermal lymphatic channel. Reprinted from Kleer CG, van Golen KL, Braun T, Merajver SD. PersistentE-cadherin expression in inflammatory breast cancer. Mod Pathol 2001;14(5):458-464, with permission from Nature Publishing Group.

Recently, breast magnetic resonance imaging (MRI) has become a primary modality for the depiction of IBC disease entities, either in conjunction with or in replacement of standard mammography. MRI can be obtained in place of mammography if mammography would be too painful or uncomfortable for the patient or if compression is inadequate secondary to tumor bulk. Although patients are usually evaluated initially with mammography, MRI is useful for determining the extent of disease and may be helpful in monitoring treatment response in some cases.

Global skin thickening, enhancing masses, breast enlargement, breast/chest wall edema, and/or tumor infiltration is often obscured on mammography. However, these entities are well characterized on MRI, leading to a higher detection rate of primary breast parenchymal lesions/masses using this modality. Multiple enhancing breast masses are more often described 
on MRI than a single index breast lesion in IBC (1). Primary tumors in IBC often demonstrate low signal intensity on T1WI and heterogeneous iso- or high signal intensity on T2WI. Increased signal intensity and enhancement of the thickened skin are also consistently reported attributes of IBC (2).

Inflammatory breast carcinoma is not the only disease process that presents with mammographic or physical signs of breast tissue inflammation. In fact, the differential for these findings remains vast, and correlation with patient history in conjunction with physical and radiologic examination findings remains crucial in these situations. Inflammation of the breast tissue may be seen after surgery and postradiation therapy, as well as from trauma, infection (i.e., mastitis), and various dermatologic disorders. Breast edema may be secondary to other systemic conditions such as superior vena cava syndrome, congestive heart failure, and lymphoma. In patients who present with inflammatory signs that continue despite proper antibiotic treatment, biopsy is indicated to definitively exclude a diagnosis of IBC (3).

Goldfarb and Pippen recently reviewed treatment of IBC at Baylor University Medical Center at Dallas (8). As they noted, the recommended therapy is induction chemotherapy with an anthracycline-based regimen with or without the addition of taxanes, followed by mastectomy with axillary lymph node dissection in those who respond well. Preoperative trastuzumab may be considered for patients with HER2-positive disease. Any remaining cycles of planned chemotherapy can be completed after surgery, and patients with hormone receptor-positive disease may begin endocrine therapy after surgery as well. Postsurgical radiotherapy of the chest wall and regional axillary nodes should follow completion of the chemotherapy regimens.
The current case describes a patient who presented for her first mammogram with an advanced inflammatory breast carcinoma, undoubtedly neglected for some time given initial palpation 2 years prior. This case highlights the importance of mammography in screening for breast carcinoma and of seeking proper and timely evaluation upon palpation of a new breast mass. It is unique given the extensive inflammatory change that was denied evaluation until a late and advanced stage. Although IBC can easily be mistaken for an infection or other disease process as discussed previously, failure to improve with antibiotics/treatment should remain a "red flag" for the relatively rare diagnosis of IBC.

1. Le-Petross HT, Cristofanilli M, Carkaci S, Krishnamurthy S, Jackson EF, Harrell RK, Reed BJ, Yang WT. MRI features of inflammatory breast cancer. AJR Am J Roentgenol 2011;197(4):W769-W776.

2. Lee KW, Chung SY, Yang I, Kim HD, Shin SJ, Kim JE, Chung BW, Choi JA. Inflammatory breast cancer: imaging findings. Clin Imaging 2005;29(1):22-25.

3. Günhan-Bilgen I, Ustün EE, Memiş A. Inflammatory breast carcinoma: mammographic, ultrasonographic, clinical, and pathologic findings in 142 cases. Radiology 2002;223(3):829-838.

4. Anderson WF, Schairer C, Chen BE, Hance KW, Levine PH. Epidemiology of inflammatory breast cancer (IBC). Breast Dis 2005-2006;22:923.

5. Kushwaha AC, Whitman GJ, Stelling CB, Cristofanilli M, Buzdar AU. Primary inflammatory carcinoma of the breast: retrospective review of mammographic findings. AJR Am J Roentgenol 2000;174(2):535-538.

6. Yang WT. Advances in imaging of inflammatory breast cancer. Cancer 2010;116(11 Suppl):2755-2757.

7. Taylor GW, Meltzer A. "Inflammatory carcinoma" of the breast. Am J Cancer 1938;33:33-49.

8. Goldfarb JM, Pippen JE. Inflammatory breast cancer: the experience of Baylor University Medical Center at Dallas. Proc (Bayl Univ Med Cent) 2011;24(2):86-88. 